\title{
Torque Ripple Suppression Control for PMSMs using Feedforward Compensation and Online Parameter Estimation
}

\author{
Noriya Nakao*a) \\ Member, \\ Kazuaki Tobari* \\ Member \\ Tomohiro Sugino** \\ Member, \\ Yoshiki Ito** \\ Member \\ Mitsuhiro Mishima** Member, \\ Daisuke Maeda** \\ Member
}

J-STAGE Advance published date : May 21, 2021

\begin{abstract}
This paper presents a control scheme for smooth torque production in permanent-magnet synchronous motor drives. The proposed control scheme consists of two techniques: a feedforward compensation for torque ripple minimization and an online parameter estimation technique for harmonic fluxes. The feedforward compensation is constructed on a position-sensorless vector control system and generates voltage commands to suppress torque ripples. The command calculation process requires the parameters of the harmonic fluxes; therefore, the proposed control scheme includes their estimator and calculates them from detected current information. These techniques are simpler than existing ones and can be implemented on a computational device with a small amount of calculation load. The proposed control scheme is verified by performing simulations and experiments.
\end{abstract}

Keywords: permanent-magnet synchronous motor, torque ripple, feedforward compensation, parameter estimation

\section{Introduction}

Permanent Magnet Synchronous Motors (PMSM) are drive sources of high output density and efficiency that are widely used for both commercial and industrial purposes. It is known that with PMSM, spatial harmonics caused by the motor's shape generate torque ripples. Depending on the driving conditions of the motor, the torque ripples can excite mechanical resonance and, consequently, cause vibration and noise or fluctuations in the motor speed.

Torque ripples can be reduced by optimizing the motor's structural design or by generating compensation signals through control. Several effective techniques related to the former have been presented, involving optimization of the magnet shape, the magnetization method, or the number of poles and slots ${ }^{(1)}$. Many techniques latter have been proposed as well; they are based on the detection/estimation of torque ripples, followed by the generation of compensation signals ${ }^{(2)-(7)}$ based on those results. In principle, this is a general-purpose approach that can be applied to any motor. For this reason, control-based torque ripple suppression (hereinafter referred to as "torque ripple suppression control") is more suitable for devices to which an unspecified motor is connected.

Torque ripple suppression control requires a means to detect/estimate torque ripples and a means to generate compensation signals based on those results. Some methods use

a) Correspondence to: Noriya Nakao. E-mail: noriya.nakao.be@ hitachi.com

* Research \& Development Group, Hitachi, Ltd.

7-1-1, Omika-cho, Hitachi, Ibaraki 319-1292, Japan

** Hitachi Power Semiconductor Device, Ltd.

5-2-2, Omika-cho, Hitachi, Ibaraki 319-1221, Japan a torque detector to detect torque ripples, when it is possible to install additional sensors ${ }^{(2)(3)}$. There is also a technique that detects torque ripples indirectly through the vibrations detected by an acceleration sensor installed in the motor frame ${ }^{(4)}$. Methods that use existing sensors (e.g., current sensor) include the technique that uses the information of detected current and voltage commands to estimate the harmonic components of induced voltage using an observer and then calculates the torque ripples based on that information and a mathematical model ${ }^{(5)}$. There are also precise torque equations that are said to be able to estimate torque ripples with only existing sensor information ${ }^{(6)}$. Further, another technique proposes estimating torque ripples based on speed sensor information ${ }^{(7)}$.

The technique that generates compensation signals generally generates current commands required to smooth the torque based on the torque ripple detection/estimation results and controls the current. In such cases, the current commands generated are signals that change according to the torque ripples, which requires a highly responsive current control. Various effective techniques based on this principle have been proposed $^{(5)(8)}$.

As described above, many means of torque ripple suppression control have been proposed so far, but the implementation of these control schemes requires a computational device with an adequate capability, and in the case of low-cost motor driving devices, in particular, it is not easy to implement a new control function. Therefore, in this paper, we propose a control scheme that realizes a torque ripple suppression control with a small calculation load. This scheme has the following characteristics: (1) it gives compensation signals (voltage command) as feedforward to generate current to suppress torque ripples and (2) extracts ripple components 
contained in the detected current and estimates distortion components of the magnetic flux (the cause of torque ripples) based on that information. The proposed control scheme based on (1) and (2) can operate using only already-equipped sensors. Also, it features a function that partially lowers the calculation frequency so that it can be implemented on a lowcost 16-bit microcomputer. Below, we detail the mathematical model of PMSMs and the basic configuration of the control system in Chapters 2 and 3, and how (1) and (2) were implemented in Chapter 4. In Chapter 5, we verify the effectiveness of the proposed control scheme based on simulation and experiment results.

\section{Mathematical Model of PMSMs Considering Spatial Harmonics}

Figure 1 shows the definition of the coordinate system. In this paper, we used the d-q coordinate system, which indicates the rotor's actual phase, and the dc-qc coordinate system, which indicates the rotor's virtual phase in the control. The $\mathrm{N}$ pole direction of the magnetic flux is defined as the daxis, and the direction rotated $\pi / 2$ counterclockwise from the $\mathrm{d}$-axis as the q-axis. The phase of the d-axis seen from the $\mathrm{U}$-axis (which indicates the U-phase direction of the stator) is defined as $\theta_{d}$, and the rotational angular speed as $(P / 2) \omega_{r}$. Also, in the dc-qc coordinate system, the phase of the dcaxis seen from the $\mathrm{U}$-axis is defined as $\theta_{d c}$, and the rotational angular speed as $\omega_{1}$.

The voltage equation of PMSMs is represented by Eq. (1):

$$
\left[\begin{array}{l}
v_{d} \\
v_{q}
\end{array}\right]=R \cdot\left[\begin{array}{l}
i_{d} \\
i_{q}
\end{array}\right]+s \cdot\left[\begin{array}{l}
L_{d} \cdot i_{d} \\
L_{q} \cdot i_{q}
\end{array}\right]+\left[\begin{array}{l}
e_{d} \\
e_{q}
\end{array}\right] \ldots \ldots \ldots \ldots \ldots \ldots \ldots \ldots \ldots(1)
$$

Here, $R$ : winding resistance; $L_{d}, L_{q}$ : inductance on $\mathrm{d}$-q coordinates; $v_{d}, v_{q}$ : stator voltage on d-q coordinates; $i_{d}, i_{q}$ : stator current on d-q coordinates; $e_{d}$, $e_{q}$ : induced voltage on d-q coordinates; $s$ : derivative operator

If the harmonic components (spatial harmonics) in the spatial distribution of magnetic fluxes are considered, the induced voltage $e_{d}, e_{q}$ is represented by Eqs. (2)-(7):

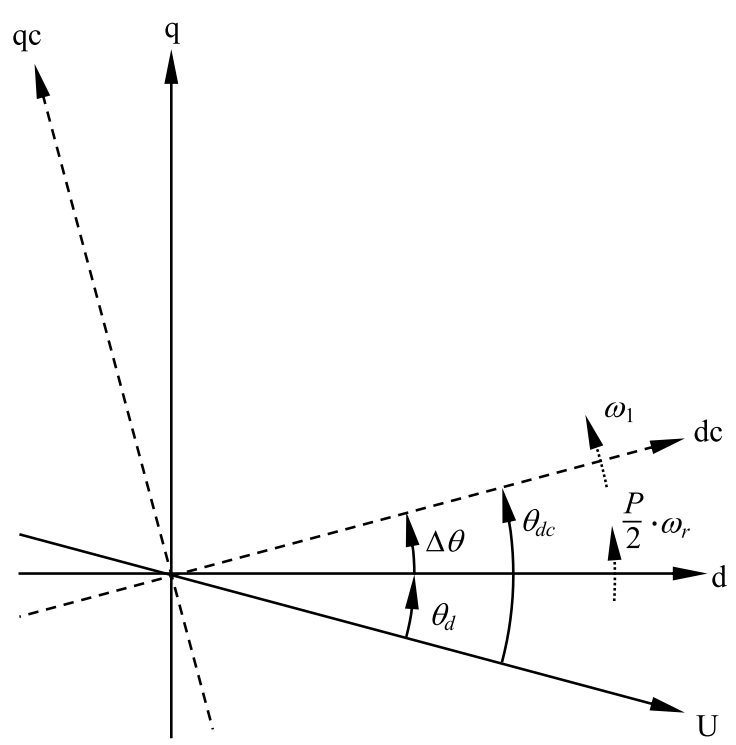

Fig. 1. Definition of reference frames

$$
\begin{aligned}
& {\left[\begin{array}{l}
e_{d} \\
e_{q}
\end{array}\right]=\frac{P}{2} \omega_{r} \cdot \boldsymbol{J} \cdot\left[\begin{array}{l}
\phi_{d} \\
\phi_{q}
\end{array}\right] \ldots \ldots \ldots \ldots} \\
& {\left[\begin{array}{l}
\phi_{d} \\
\phi_{q}
\end{array}\right]=\left[\begin{array}{l}
\phi_{i d}+\phi_{m d} \\
\phi_{i q}+\phi_{m q}
\end{array}\right] \ldots \ldots \ldots \ldots \ldots} \\
& {\left[\begin{array}{l}
\phi_{i d} \\
\phi_{i q}
\end{array}\right]=\left[\begin{array}{l}
L_{d} \cdot i_{d} \\
L_{q} \cdot i_{q}
\end{array}\right] \ldots \ldots \ldots \ldots \ldots} \\
& {\left[\begin{array}{l}
\phi_{m d} \\
\phi_{m q}
\end{array}\right]=\left[\begin{array}{c}
\Phi_{m 1} \\
0
\end{array}\right]+\left[\begin{array}{l}
\Phi_{m h d} \cdot \cos 6 \theta_{d} \\
\Phi_{m h q} \cdot \sin 6 \theta_{d}
\end{array}\right]} \\
& {\left[\begin{array}{l}
\Phi_{m h d} \\
\Phi_{m h q}
\end{array}\right]=\left[\begin{array}{c}
\Phi_{m 5}+\Phi_{m 7} \\
-\Phi_{m 5}+\Phi_{m 7}
\end{array}\right] \ldots \ldots \ldots} \\
& \boldsymbol{J}=\left[\begin{array}{cc}
0 & -1 \\
1 & 0
\end{array}\right] \ldots \ldots \ldots \ldots \ldots \ldots
\end{aligned}
$$

Here, $P$ : the number of poles in the motor; $\phi_{d}, \phi_{q}$ : all interlinkage fluxes of the stator on d-q coordinates; $\phi_{i d}, \phi_{i q}$ : stator interlinkage fluxes generated with the current on d-q coordinates; $\phi_{m d}, \phi_{m q}$ : interlinkage fluxes of the stator generated by permanent magnets on d-q coordinates; $\Phi_{m 1}$ : amplitude value of magnetic flux fundamental components; $\Phi_{m h d}, \Phi_{m h q}$ : amplitude value of magnetic flux harmonic components on d-q coordinates; $\Phi_{m 5}$ : amplitude value of magnetic flux fifth-order harmonic components; $\Phi_{m 7}$ : amplitude value of magnetic flux seventh-order harmonic components

The torque equation of PMSMs is represented by Eq. (8):

$$
\begin{aligned}
\tau= & \frac{3 P}{4}\left(\phi_{d} i_{q}-\phi_{q} i_{d}\right) \\
= & \frac{3 P}{4}\left\{\left(L_{d}-L_{q}\right) i_{d} i_{q}+\Phi_{m 1} i_{q}\right. \\
& \left.+\Phi_{m h d} i_{q} \cdot \cos 6 \theta_{d}-\Phi_{m h q} i_{d} \cdot \sin 6 \theta_{d}\right\} \ldots \ldots .
\end{aligned}
$$

The first and second terms of the right side of Eq. (8) are respectively the active components of reluctance torque and magnet torque, and the third and fourth terms correspond to the ripple torque. The mathematical model above considers the harmonic components of magnetic flux of up to the seventh order.

\section{Basic Configuration of the Control System}

Figure 2 shows the block diagram of the control system. This system configuration is divided into the vector control unit, the position sensorless control unit, and the torque ripple suppression control unit. First, we describe the vector control and position sensorless control units constructed based on the technique detailed in Ref. (9); then, we explain the details of the proposal, the torque ripple suppression control unit.

In the vector control unit, instead of speed and current control by feedback control, a control that gives feedforward voltage command is performed. This limits the control responsiveness, but it makes it possible to reduce the number of spots in the control design that require adjustments. The voltage command by vector control, $V_{d}{ }^{*}$ and $V_{q}{ }^{*}$, is represented by Eq. (9):

$$
\left[\begin{array}{c}
V_{d}{ }^{*} \\
V_{q}{ }^{*}
\end{array}\right]=R^{*} \cdot\left[\begin{array}{c}
I_{d}{ }^{*} \\
I_{q}{ }^{*}
\end{array}\right]+\omega_{1}{ }^{*} \cdot\left[\begin{array}{c}
-L_{q}{ }^{*} \cdot I_{q}{ }^{*} \\
L_{d}{ }^{*} \cdot I_{d}{ }^{*}
\end{array}\right]+\omega_{1}{ }^{*} \cdot\left[\begin{array}{c}
0 \\
\Phi_{m 1}{ }^{*}
\end{array}\right]
$$




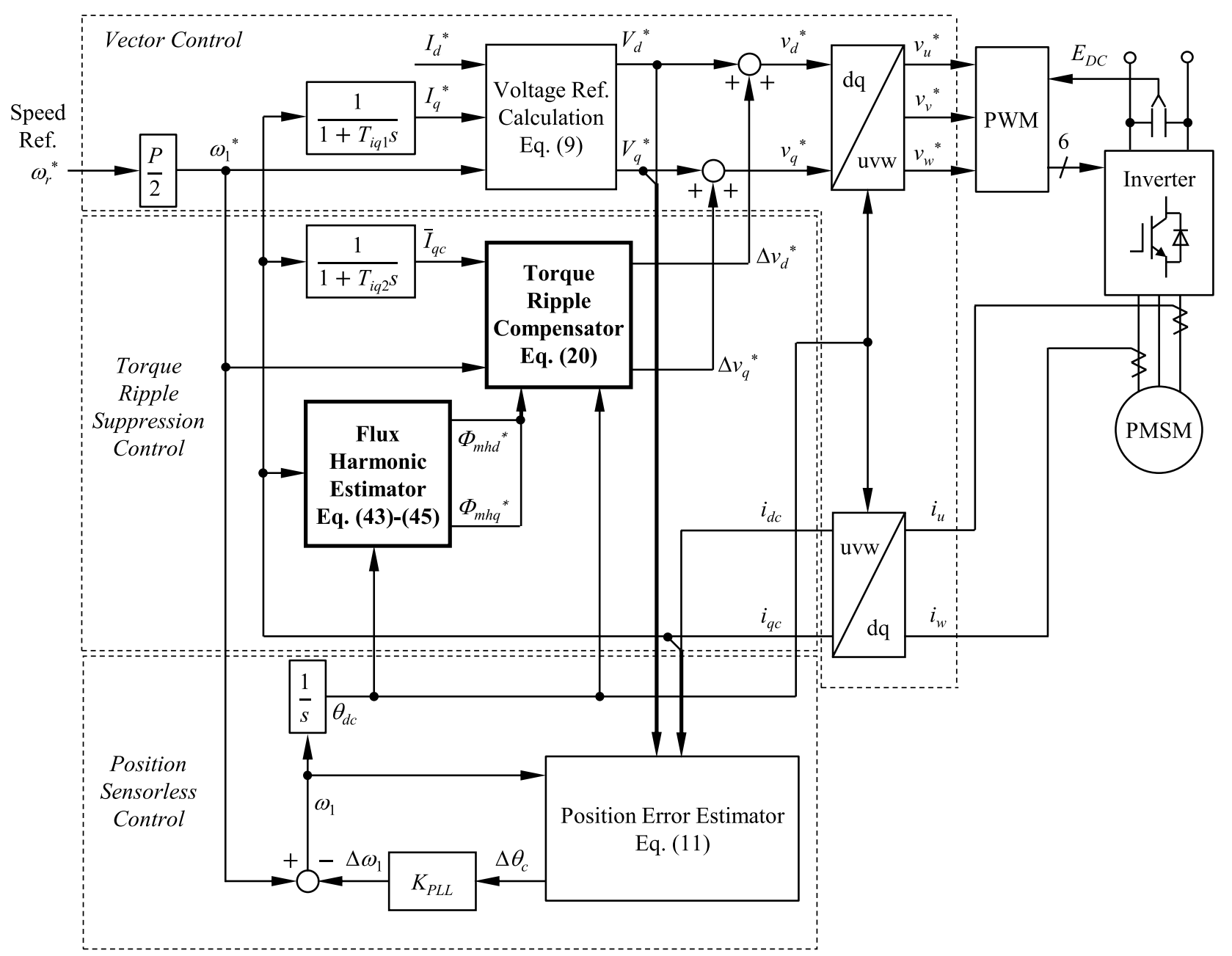

Fig. 2. Block diagram of the proposed control scheme

Here, $L_{d}{ }^{*}, L_{q}{ }^{*}$ : set value of the inductance of daxis and q-axis; $R^{*}$ : set value of winding resistance; $\Phi_{m 1}{ }^{*}$ : set value of magnetic flux fundamental components; $\omega_{1}{ }^{*}$ : inverter drive frequency command; $I_{d}{ }^{*}, I_{q}{ }^{*}$ : current commands on d-axis and q-axis

The current commands on q-axis, $I_{q}{ }^{*}$, is given by Eq. (10):

$$
I_{q}^{*}=\frac{1}{1+T_{i q 1} s} i_{q c}
$$

Here, $i_{q c}$ : detected current on q-axis; $T_{i q 1}$ : time constant of the filter for generation of current commands

On the q-axis detected current $i_{q c}$, it generates the q-axis current command $I_{q}{ }^{*}$ through the low-pass filter, and this is used as a substitute for the conventional speed control. In this paper, the d-axis current command, $I_{d}{ }^{*}$, was set to zero.

The estimated phase difference $\Delta \theta$ between d-q and dc-qc coordinate systems, $\Delta \theta_{c}$, is represented by Eq. (11):

$$
\Delta \theta_{c}=\tan ^{-1}\left(\frac{V_{d}{ }^{*}-R^{*} \cdot i_{d c}+\omega_{1} L_{q}{ }^{*} \cdot i_{q c}}{V_{q}{ }^{*}-R^{*} \cdot i_{q c}-\omega_{1} L_{q}{ }^{*} \cdot i_{d c}}\right) \ldots
$$

Here, $i_{d c}$ : d-axis detected current

The inverter drive frequency $\omega_{1}$ used in the calculation of Eq. (11) is given by Eq. (12):

$$
\omega_{1}=\omega_{1}^{*}-K_{P L L} \cdot \Delta \theta_{c}
$$

Here, $K_{P L L}$ : proportional gain of PLL control Also, the dc-axis phase is given by Eq. (13):

$$
\theta_{d c}=\frac{1}{s} \omega_{1}
$$

\section{Torque Ripple Suppression Control}

4.1 Principle of Torque Ripple Compensation

Normally, the vector control unit performs the control so that the d-axis current, $i_{d}$, and q-axis current, $i_{\mathrm{q}}$, become constant. The flowing current of this moment, if the d-axis current $i_{d}$ is set to zero, is represented by Eq. (14):

$$
\left[\begin{array}{l}
i_{d} \\
i_{q}
\end{array}\right]=\left[\begin{array}{l}
0 \\
\bar{I}_{q}
\end{array}\right]
$$

Here, $\bar{I}_{q}$ : the average value of q-axis current $i_{q}$ By substituting Eq. (14) into Eq. (8), we obtain Eq. (15):

$$
\tau=\frac{3 P}{4}\left(\Phi_{m 1} \bar{I}_{q}+\Phi_{m h d} \bar{I}_{q} \cdot \cos 6 \theta_{d}\right)
$$

The first and second terms of the right side of Eq. (15) respectively correspond to the effective torque and rippling torque. To compensate torque ripples, it is necessary to generate a ripple current with an inverted phase to the fluctuating torque. The current for smoothing the torque is represented by Eq. (16): 


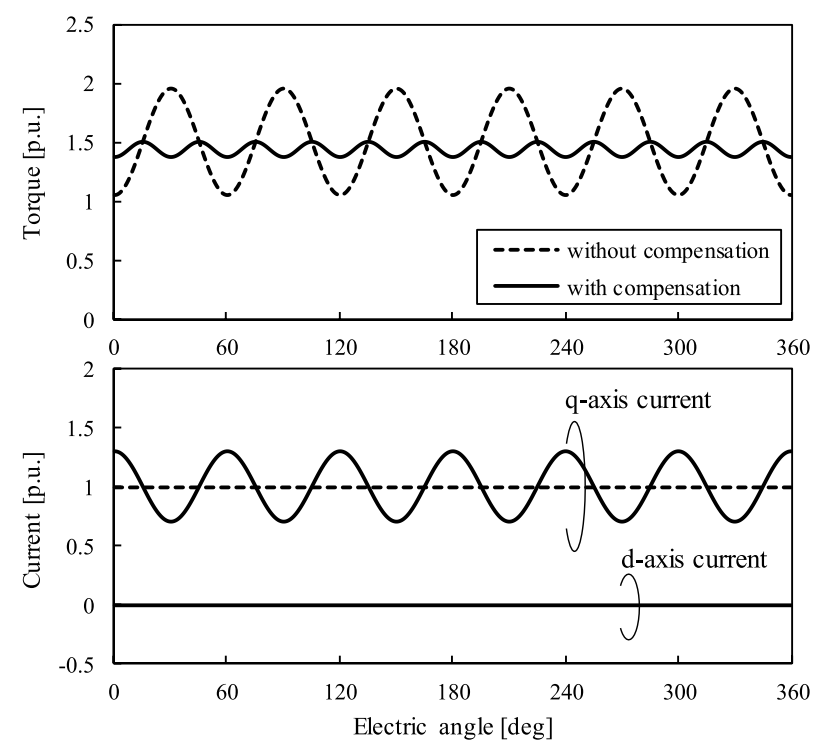

Fig. 3. Principle of torque ripple suppression

$$
\left[\begin{array}{c}
i_{d} \\
i_{q}
\end{array}\right]=\left[\begin{array}{c}
0 \\
\bar{I}_{q}+i_{q C O M P}
\end{array}\right]
$$

Here, $i_{q \text { СОMP }}$ : the ripple current, given by the following equation:

$$
i_{q \text { СOMP }}=-\frac{\Phi_{m h d}}{\Phi_{m 1}} \bar{I}_{q} \cdot \cos 6 \theta_{d} .
$$

If Eqs. (16) and (17) are substituted into Eq. (8), Eq. (18) is obtained:

$$
\tau=\frac{3 P}{4}\left(\Phi_{m 1} \bar{I}_{q}-\frac{\Phi_{m h d^{2}}}{2 \Phi_{m 1}} \bar{I}_{q}-\frac{\Phi_{m h d^{2}}}{2 \Phi_{m 1}} \bar{I}_{q} \cdot \cos 12 \theta_{d}\right)
$$

If we compare Eqs. (15) and (18), it is possible to see that the order of the ripple torque changed from sixth to twelfth, and its amplitude value reduced at the ratio of " $\Phi_{m h d} /\left(2 \Phi_{m 1}\right)$ ".

Figure 3 is an example of behavior when the currents indicated in Eqs. (14) and (16) are applied. In this case, the amplitude value of magnetic flux fundamental components $\Phi_{m 1}$ was defined as the standard (1.0), and the amplitude value of fifth-order harmonic components, $\Phi_{m 5}$, and seventh-order harmonic components, $\Phi_{m 7}$, were respectively set to 0.5 and 0.2 . Also, to normalize the torque and current, the average value of the q-axis current $i_{q}\left(\bar{I}_{q}\right)$ was set to 1 , and the number of poles in the motor $(P)$, to 2 . The dotted line of the same chart, which corresponds to Eq. (14), shows that when constant d-axis and q-axis currents are supplied, torque ripples of sixth order were generated. On the other hand, from the solid line of the graph, which corresponds to Eq. (16), it is possible to see that a fluctuating ripple current of sixth order is superimposed on the q-axis current, and a smoothed torque is generated. This is the principle of torque ripple compensation.

4.2 Torque Ripple Compensation by Feedforward Control To generate the ripple current $i_{q C O M P}$, which is a sixth-order harmonic component, the control scheme design must be able to ensure an acceptable level of current control responsiveness. However, the current control by the conventional feedback control has a trade-off between responsiveness and stability. While it is possible to achieve responsiveness and stability using a high-response control mentioned in Refs. (5) and (8), if a low-cost motor driving device is used, it may be difficult to implement a new control function. For this reason, in this paper, we propose an easier type of control that generates the ripple current $i_{q \text { СOMP }}$ by giving voltage commands in a feedforward manner.

If " $i_{d}=0, i_{q}=i_{q \text { СомP }}$ " in Eq. (1) and the first term of the right side of Eq. (5) is ignored, $\Delta v_{d}$ and $\Delta v_{q}$, the voltage required to supply the ripple current $i_{q \text { COMP }}$ is represented by Eq. (19):

$$
\begin{aligned}
& {\left[\begin{array}{l}
\Delta v_{d} \\
\Delta v_{q}
\end{array}\right]=R \cdot\left[\begin{array}{c}
0 \\
i_{q C O M P}
\end{array}\right]+s \cdot\left[\begin{array}{c}
0 \\
L_{q} \cdot i_{q C O M P}
\end{array}\right]} \\
& +\frac{P}{2} \omega_{r} \cdot \boldsymbol{J} \cdot\left[\begin{array}{c}
0 \\
L_{q} \cdot i_{q C O M P}
\end{array}\right]+\frac{P}{2} \omega_{r} \cdot \boldsymbol{J} \cdot\left[\begin{array}{l}
\Phi_{m h d} \cdot \cos 6 \theta_{d} \\
\Phi_{m h q} \cdot \sin 6 \theta_{d}
\end{array}\right] \\
& =\frac{P}{2} \omega_{r} \cdot\left[\begin{array}{cc}
-\Phi_{m h q} & L_{q} \cdot \frac{\Phi_{m h d}}{\Phi_{m 1}} \cdot \bar{I}_{q} \\
6 L_{q} \cdot \frac{\Phi_{m h d}}{\Phi_{m 1}} \cdot \bar{I}_{q} & -\frac{R}{\frac{P}{2} \omega_{r}} \cdot \frac{\Phi_{m h d}}{\Phi_{m 1}} \cdot \bar{I}_{q}+\Phi_{m h d}
\end{array}\right] \\
& \cdot\left[\begin{array}{c}
\sin 6 \theta_{d} \\
\cos 6 \theta_{d}
\end{array}\right]
\end{aligned}
$$

Based on Eq. (19), the voltage commands that generate the ripple current $i_{q C O M P}, \Delta v_{d}{ }^{*}$ and $\Delta v_{q}{ }^{*}$, are represented by Eq. (20):

$$
\begin{aligned}
& {\left[\begin{array}{l}
\Delta v_{d}{ }^{*} \\
\Delta v_{q}{ }^{*}
\end{array}\right]=\omega_{1}{ }^{*} \cdot\left[\begin{array}{cc}
-\Phi_{m h q}{ }^{*} & L_{q}{ }^{*} \cdot \frac{\Phi_{m h d^{*}}}{\Phi_{m 1}{ }^{*}} \cdot \bar{I}_{q c} \\
6 L_{q}{ }^{*} \cdot \frac{\Phi_{m h d^{*}}}{\Phi_{m 1}{ }^{*}} \cdot \bar{I}_{q c} & -\frac{R^{*}}{\omega_{1}{ }^{*}} \cdot \frac{\Phi_{m h d^{*}}}{\Phi_{m 1}{ }^{*}} \cdot \bar{I}_{q c}+\Phi_{m h d}{ }^{*}
\end{array}\right]} \\
& \text { - }\left[\begin{array}{c}
\sin 6 \theta_{d c} \\
\cos 6 \theta_{d c}
\end{array}\right] \text {. }
\end{aligned}
$$

Here, $\Phi_{m h d^{*}}$ : set value of the d-axis magnetic flux harmonic components, $\Phi_{m h q}{ }^{*}$ : set value of q-axis magnetic flux harmonic components, $\bar{I}_{q c}$ : average value of q-axis detected current $i_{q c}$

The average value of the q-axis detected current, $\bar{I}_{q c}$, is given by Eq. (21):

$$
\bar{I}_{q c}=\frac{1}{1+T_{i q 2} s} i_{q c}
$$

Here, $T_{i q 2}$ : time constant of the filter for average current calculation

The time constant of the filter for current calculation, $T_{i q 2}$, is designed so as to remove the sixth and twelfth-order harmonic components contained in the q-axis detected current $i_{q c}$. The voltage commands $\Delta v_{d}{ }^{*}$ and $\Delta v_{q}{ }^{*}$ are added to the vector- controlled voltage commands $V_{d}{ }^{*}$ and $V_{q}{ }^{*}$ and, ultimately, the motor is driven by the voltage commands $v_{d}{ }^{*}$ and $v_{q}{ }^{*}$ shown in Eq. (22):

$$
\left[\begin{array}{c}
v_{d}^{*} \\
v_{q}^{*}
\end{array}\right]=\left[\begin{array}{c}
V_{d}^{*} \\
V_{q}^{*}
\end{array}\right]+\left[\begin{array}{l}
\Delta v_{d}^{*} \\
\Delta v_{q}^{*}
\end{array}\right]
$$

As shown in Eq. (20) above, by setting a configuration that gives voltage commands $\Delta v_{d}{ }^{*}$ and $\Delta v_{q}{ }^{*}$ in a feedforward manner, it is possible to generate the ripple current $i_{q \text { СОМP }}$ 
without delays. Also, given that the amplitude value of $\Delta v_{d}{ }^{*}$ and $\Delta v_{q}{ }^{*}$ becomes constant while in steady-state, it is possible to reduce the load on the computational device by reducing the calculation frequency. For this paper, we adopted a 16-bit microcomputer and set a low-speed calculation unit that operates in 1-ms cycles and a high-speed calculation unit that operates in $187.5 \mu$ s cycles (equivalent to three cycles of carrier frequency $16 \mathrm{kHz}$ ). The calculation frequency of the high-speed calculation unit is about 5.33 times that of the low-speed calculation unit $(1 \mathrm{~ms} / 187.5 \mu \mathrm{s} \approx 5.33)$. We also implemented a control software on the microcomputer so that the amplitude value of voltage commands $\Delta v_{d}{ }^{*}$ and $\Delta v_{q}{ }^{*}$ is calculated at the low-speed calculation unit, and the multiplication of $\sin 6 \theta_{d c}$ and $\cos 6 \theta_{d c}$ at the high-speed calculation unit. With this, it is possible to reduce the processing load to about $19 \%(1 / 5.33=0.188)$ compared to if all amplitude value calculations were processed at the high-speed calculation unit.

4.3 Estimation of Magnetic Flux Harmonic Components by Current Detection As shown in Eq. (20), the set value of each parameter was used to calculate the voltage commands $\Delta v_{d}{ }^{*}$ and $\Delta v_{q}{ }^{*}$, and it is necessary to set these values without errors. The set value of $\mathrm{d}$-axis and q-axis magnetic flux harmonic components- $\Phi_{m h d}{ }^{*}$ and $\Phi_{m h q}{ }^{*}$ - in particular, are important parameters that determine the torque ripple reduction effect. While it is possible to obtain these in advance with preliminary tests, it is preferable to estimate them online based on sensor information because of its higher versatility. Therefore, in this paper, we present a method to estimate the amplitude value of magnetic flux harmonic components on d-q coordinates, $\Phi_{m h d}$ and $\Phi_{m h q}$, based on the detected current.

By solving Eq. (1) for d-axis and q-axis currents, $i_{d}$ and $i_{q}$, we obtain Eq. (23):

$$
\left[\begin{array}{c}
i_{d} \\
i_{q}
\end{array}\right]=\left[\begin{array}{c}
\frac{1}{R+L_{d} \cdot s} \cdot\left\{v_{d}+\frac{P}{2} \omega_{r} \cdot\left(L_{q} \cdot i_{q}+\Phi_{m h q} \cdot \sin 6 \theta_{d}\right)\right\} \\
\frac{1}{R+L_{q} \cdot s} \cdot\left\{v_{q}-\frac{P}{2} \omega_{r} \cdot\left(L_{d} \cdot i_{d}+\Phi_{m 1}+\Phi_{m h d} \cdot \cos 6 \theta_{d}\right)\right\}
\end{array}\right]
$$

If the result obtained by substituting Eqs. (9) and (20) into Eq. (22) is substituted into Eq. (23) as " $v_{d}=v_{d}{ }^{*}$ and $v_{q}=v_{q}{ }^{*}$ ", Eqs. (24)-(30) are obtained:

$$
\begin{aligned}
& {\left[\begin{array}{l}
i_{d} \\
i_{q}
\end{array}\right]=\left[\begin{array}{l}
0 \\
\bar{I}_{q}
\end{array}\right]+\left[\begin{array}{ll}
\Delta I_{d 1} & \Delta I_{d 2} \\
\Delta I_{q 1} & \Delta I_{q 2}
\end{array}\right] \cdot\left[\begin{array}{c}
\sin 6 \theta_{d} \\
\cos 6 \theta_{d}
\end{array}\right] \ldots \ldots \ldots \ldots} \\
& \Delta I_{d 1}=\frac{1}{6 L_{d}} \cdot\left(L_{q} \cdot \frac{\Phi_{m h d^{*}}}{\Phi_{m 1}} \cdot \bar{I}_{q}+L_{q} \cdot \Delta I_{q 2}\right) \ldots \ldots \ldots \\
& \Delta I_{d 2}=-\frac{1}{6 L_{d}} \cdot\left(\Delta \Phi_{m h q}+L_{q} \cdot \Delta I_{q 1}\right) \ldots \ldots \ldots \ldots \\
& \Delta I_{q 1}=-\frac{1}{6 L_{q}} \cdot\left(\Delta \Phi_{m h d}+L_{d} \cdot \Delta I_{d 2}\right) \ldots \ldots \ldots \ldots \\
& \Delta I_{q 2}=-\frac{1}{6 L_{q}} \cdot\left(6 L_{q} \cdot \frac{\Phi_{m h d}{ }^{*}}{\Phi_{m 1}} \cdot \bar{I}_{q}-L_{d} \cdot \Delta I_{d 1}\right) \ldots \ldots \\
& \Delta \Phi_{m h d}=\Phi_{m h d}-\Phi_{m h d}{ }^{*} \ldots \ldots \ldots \ldots \ldots \ldots \\
& \Delta \Phi_{m h q}=\Phi_{m h q}-\Phi_{m h q}{ }^{*} \ldots \ldots \ldots \ldots \ldots \ldots
\end{aligned}
$$

Here, except for the set value of the d-axis and q-axis magnetic flux harmonic components, $\Phi_{m h d}{ }^{*}$ and $\Phi_{m h q}{ }^{*}$, there is no error in the set value of the motor parameters, and we assumed that the phase difference $\Delta \theta$ is zero. Eqs. (31)-(34) below were also applied:

$$
\begin{aligned}
& \bar{I}_{d}=I_{d}{ }^{*}=0 \\
& \bar{I}_{q}=I_{q}{ }^{*}=\bar{I}_{q c} \\
& \frac{P}{2} \omega_{r}=\omega_{1}{ }^{*} \\
& 0 \ll \omega_{r} \cdots
\end{aligned}
$$

From Eqs. (26) and (27), Eqs. (35) and (36) are obtained:

$$
\begin{aligned}
\Delta I_{d 2} & =\left(1-\frac{1}{6^{2}}\right)^{-1} \cdot\left(-\frac{1}{6 L_{d}} \cdot \Delta \Phi_{m h q}+\frac{1}{6^{2} L_{d}} \cdot \Delta \Phi_{m h d}\right) \\
& \approx-\frac{1}{6 L_{d}} \cdot \Delta \Phi_{m h q} \ldots \ldots \ldots \ldots \ldots \ldots \ldots \ldots(35) \\
\Delta I_{q 1} & =-\frac{1}{6 L_{q}} \cdot \Delta \Phi_{m h d}+\frac{1}{6^{2} L_{q}} \cdot \Delta \Phi_{m h q} \\
& \approx-\frac{1}{6 L_{q}} \cdot \Delta \Phi_{m h d} \ldots \ldots \ldots \ldots \ldots \ldots \ldots
\end{aligned}
$$

From Eqs. (35) and (36), it is possible to see that the setting error of the q-axis magnetic flux harmonic component, $\Delta \Phi_{m h q}$, appears in the $\cos 6 \theta_{d}$ component of the d-axis current $i_{d}$, and the setting error of the d-axis magnetic flux harmonic component, $\Delta \Phi_{m h d}$, appears in the $\sin 6 \theta_{d}$ component of the q-axis current $i_{q}$. Therefore, through the integration in Eqs. (37) and (38) below, it is possible to estimate the amplitude values of magnetic flux harmonic components, $\Phi_{m h d}$ and $\Phi_{m h q}$, from a detected current.

$$
\begin{aligned}
& \widehat{\Phi}_{m h d}=K_{I \Phi d} \cdot \int \Delta I_{q 1} \cdot d t+\Phi_{m h d 0} \cdots \ldots \ldots \ldots \ldots \ldots \ldots \ldots \\
& \widehat{\Phi}_{m h q}=K_{I \Phi q} \cdot \int \Delta I_{d 2} \cdot d t+\Phi_{m h q 0} \cdots \ldots \ldots \ldots \ldots
\end{aligned}
$$

Here, $\hat{\Phi}_{m h d}$, $\hat{\Phi}_{m h q}$ : estimated values of amplitude values $\Phi_{m h d}$ and $\Phi_{m h q} ; \Phi_{m h d 0}, \Phi_{m h q 0}$ : initial values of estimated values $\Phi_{m h d}$ and $\Phi_{m h q} ; K_{I \Phi d}, K_{I \Phi q}$ : integral control gain for estimating $\Phi_{m h d}$ and $\Phi_{m h q}$

The amplitude values $\Delta I_{q 1}$ and $\Delta I_{d 2}$ are given by the filtering process shown in Eqs. (39) and (40), respectively:

$$
\begin{aligned}
\Delta I_{q 1} & =\frac{2}{1+T_{\Phi} S}\left(i_{q} \cdot \sin 6 \theta_{d}\right) . \\
\Delta I_{d 2} & =\frac{2}{1+T_{\Phi} S}\left(i_{d} \cdot \cos 6 \theta_{d}\right)
\end{aligned}
$$

Here, $T_{\Phi}$ : time constant of the filter for ripple current extraction

In Eq. (39), if the q-axis current $i_{q}$, which contains the sin $6 \theta_{d}$ component, is multiplied by the in-phase $\sin 6 \theta_{d}$ and doubled, it generates a calculation result containing the amplitude value of sixth-order harmonic components, $\Delta I_{q 1}$, and twelfth-order harmonic components. Meanwhile, by removing the twelfth-order harmonic components with the low-pass filtering process with the time constant $T_{\Phi}$, the amplitude value $\Delta I_{q 1}$ was extracted. The extraction of the amplitude value $\Delta I_{d 2}$ by Eq. (40) follows the same calculation principle. 
4.4 The Effect of Dead Time in Parameter Estimation and Its Countermeasure In the previous section, the parameter estimation indicated in Eqs. (37) and (38) was constructed assuming that a ripple current was generated by the magnetic flux harmonic components shown in the second term of Eq. (5). However, if a motor is driven by an inverter, a ripple current is also generated by dead time. For this reason, it is necessary to consider the dead-time effect to estimate the parameters accurately.

The distortion voltage generated by dead time is represented by Eq. (41):

$$
\left[\begin{array}{c}
\Delta v_{u} \\
\Delta v_{v} \\
\Delta v_{w}
\end{array}\right]=f_{c} \cdot T_{d} \cdot E_{D C} \cdot\left[\begin{array}{c}
\operatorname{sgn}\left(i_{u}\right) \\
\operatorname{sgn}\left(i_{v}\right) \\
\operatorname{sgn}\left(i_{w}\right)
\end{array}\right] \cdots \cdots \cdots \cdots \cdots \cdots \cdots \cdots
$$

Here, $\Delta v_{u}, \Delta v_{v}, \Delta v_{w}$ : voltage due to dead time on three-phase stationary coordinates; $f_{c}$ : carrier frequency; $T_{d}$ : dead time set time; $E_{D C}$ : inverter DC voltage; $i_{u}, i_{v}, i_{w}$ : stator current on three-phase stationary coordinates

Figure 4 illustrates the voltage distortion shown in Eq. (41) with d-q coordinates. However, only the q-axis current is energized, and the d-axis current is zero. That is, the q-axis and d-axis respectively correspond to the energized and nonenergized axes. The respective amplitude value in Fig. 4 indicates that the q-axis components are smaller than the d-axis components. This means that the dead-time effect that appears on the energized axis is smaller than that of the nonenergized axis. With the torque ripple suppression control proposed in this paper, only the q-axis current is energized, as shown in Eq. (16). This suggests that between Eqs. (37) and (38), the former, which uses the current on the energized axis, estimates the parameters more accurately.

Then, if we assume that the fifth-order components are the main ones among the magnetic flux harmonic components,

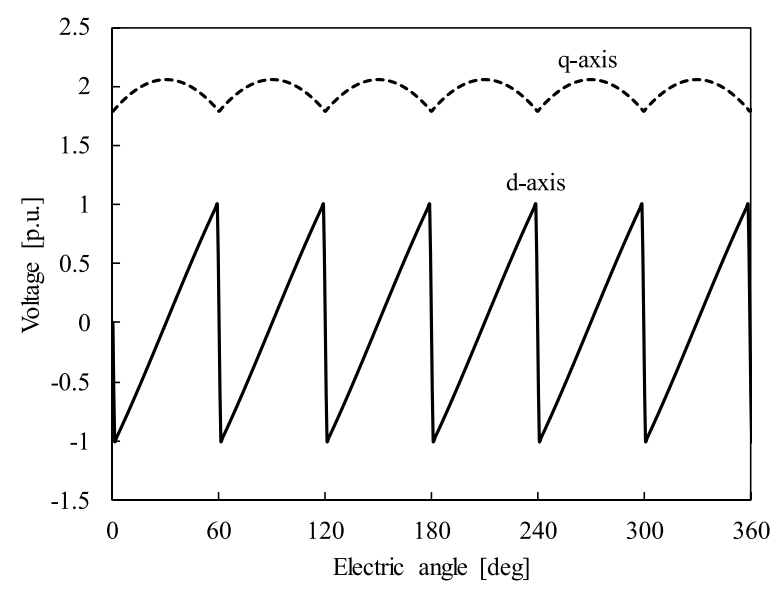

Fig. 4. Voltage distortion due to inverter dead-time

we obtain Eq. (42):

$$
\Phi_{m h d}=-\Phi_{m h q}
$$

From Eqs. (37) and (42), in this paper, the parameter estimation shown in Eq. (43) and (44) is applied.

$$
\begin{aligned}
& \Phi_{m h d}{ }^{*}=K_{I \Phi d} \cdot \int \Delta I_{q c 1} \cdot d t+\Phi_{m h 0}{ }^{*} \\
& \Phi_{m h q}{ }^{*}=-\Phi_{m h d}{ }^{*} \ldots \ldots \ldots \ldots \ldots \ldots \ldots \ldots
\end{aligned}
$$

Here, $\Delta I_{q c 1}$ : amplitude value of $\sin 6 \theta_{d c}$ components of q-axis detected current; $\Phi_{m h 0}{ }^{*}$ : initial value of set value $\Phi_{m h{ }^{*}}{ }^{*}$

Figure 5 is the block diagram of a harmonic magnetic flux estimator constructed based on Eqs. (43) and (44). This estimator is configured to estimate the amplitude values $\Phi_{m h d}$ and $\Phi_{m h q}$ based on the q-axis current. The amplitude value $\Delta I_{q c 1}$ is given based on Eq. (39) by the filtering process shown in Eq. (45):

$$
\Delta I_{q c 1}=\frac{2}{1+T_{\Phi} S}\left(i_{q c} \cdot \sin 6 \theta_{d c}\right)
$$

In steady-state, the calculation result after the filtering process in the harmonic magnetic flux estimator becomes constant. From this, if the process after integral control is implemented so that it is processed at the low-speed calculation unit of the microcomputer, it is possible to reduce the calculation load as in the calculation of Eq. (20).

\section{Simulation and Experiment}

Table 1 shows the specifications of the tested motor, a PMSM for driving the fan of an air conditioner outdoor unit.

5.1 Verification by Simulation Figure 6 shows simulation results of constant-speed operation when the deadtime effect is not considered. The load torque is $0.3375 \mathrm{Nm}$, the rotational speed is $675 \mathrm{~min}^{-1}$, and the d-axis current command $I_{d c}{ }^{*}$ is 0 A. During $0-1.0 \mathrm{~s}$, the normal vector control was applied, which was switched to torque ripple suppression control during 1.0-2.0 s. Also, magnetic flux harmonic components of up to the fifth order were considered. With this, " $\left|\Phi_{m h d}\right|=\left|\Phi_{m h q}\right| "$ holds, and Eqs. (43) and (44) have the same estimation accuracy. For this reason, only the estimation result of amplitude value $\Phi_{m h d}$ is shown. The initial value $\Phi_{m h 0}{ }^{*}$ is set to zero.

The results shown in Fig. 6 indicate that, as the set value of

Table 1. Specification of tested PMSM

\begin{tabular}{|c|c|c|c|}
\hline$P$ & 8 & $L_{q}$ & $281.7 \mathrm{mH}$ \\
\hline$R$ & $29.5 \Omega$ & $\Phi_{m 1}$ & $199 \mathrm{mVs} / \mathrm{rad}$ \\
\hline$L_{d}$ & $269.0 \mathrm{mH}$ & $\Phi_{m 5}$ & $5.11 \mathrm{mVs} / \mathrm{rad}$ \\
\hline
\end{tabular}

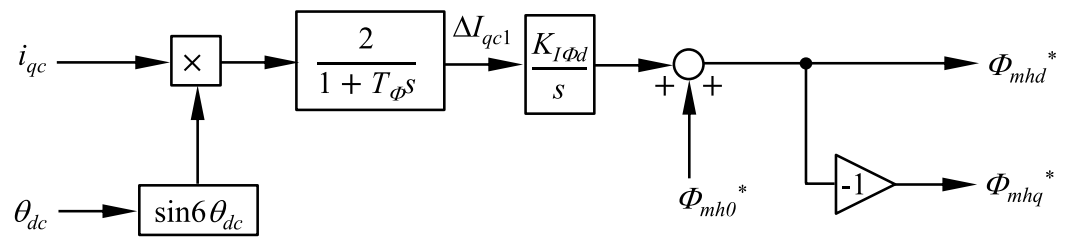

Fig. 5. Block diagram of harmonic-flux estimator 


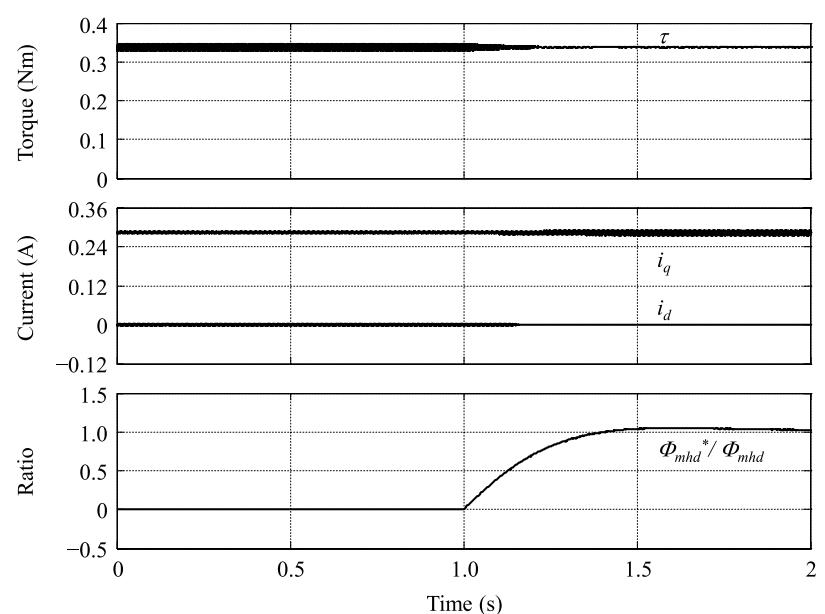

Fig. 6. Result of the proposed control scheme by simulation without consideration of dead-time effect

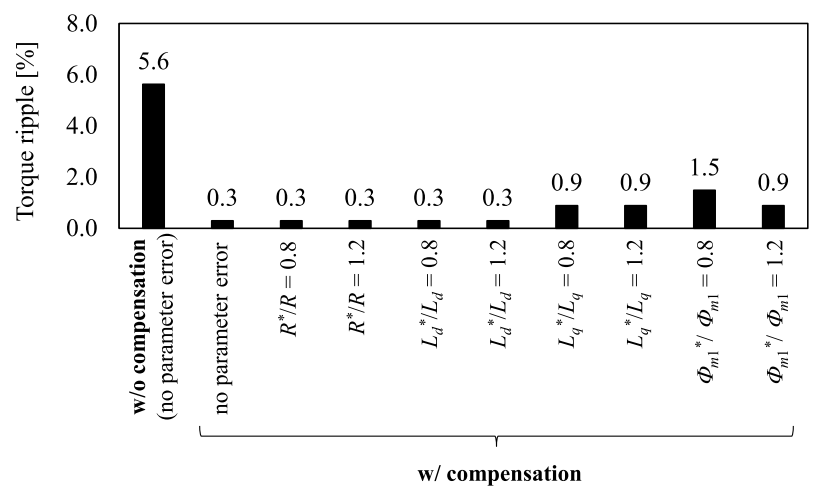

Fig. 7. Influence of parameter-setting errors on the proposed control scheme

the magnetic flux harmonic components, $\Phi_{m h d}{ }^{*}$, converges to the true value, the ripple current on d-axis is removed, and the ripple current on q-axis is superimposed. As a result, the torque ripples are effectively reduced.

Figure 7 shows the results obtained with the proposed control scheme when an error of $\pm 20 \%$ is set to each parameter. It shows that in all cases, the proposed control scheme successfully reduced torque ripples. However, when an error was set for $L_{q}{ }^{*}$ and $\Phi_{m 1}{ }^{*}$, the performance dropped quite significantly. As previously shown in Eq. (11), $L_{q}{ }^{*}$ is used in the calculation of the phase difference in the position sensorless control, $\Delta \theta_{\mathrm{c}}$. This indicates that the setting error of $L_{q}{ }^{*}$ decreases the position estimation accuracy and causes a difference in the phase of voltage commands $\Delta v_{d}{ }^{*}$ and $\Delta v_{q}{ }^{*}$ based on Eq. (20). As a result, torque ripples remain. Meanwhile, given that $\Phi_{m 1}{ }^{*}$ is a parameter that determines the amplitude value of the ripple current $i_{q C O M P}$, as shown in Eq. (17), it is evident that this setting error reduces the torque ripple reduction effect.

Next, Fig. 8 shows the results considering the dead-time effect. The other conditions are the same as those of the simulation shown in Fig. 6. Also, the control to compensate the dead time was not applied. Figure 8(a) shows the amplitude value of magnetic flux harmonic components $\Phi_{m h d}$ and $\Phi_{m h q}$ estimated with the d-axis detected current $i_{d c}$ based on Eqs. (38), (40), and (42). The results of parameter estimation show that the set value $\Phi_{m h d}{ }^{*}$ deviated significantly

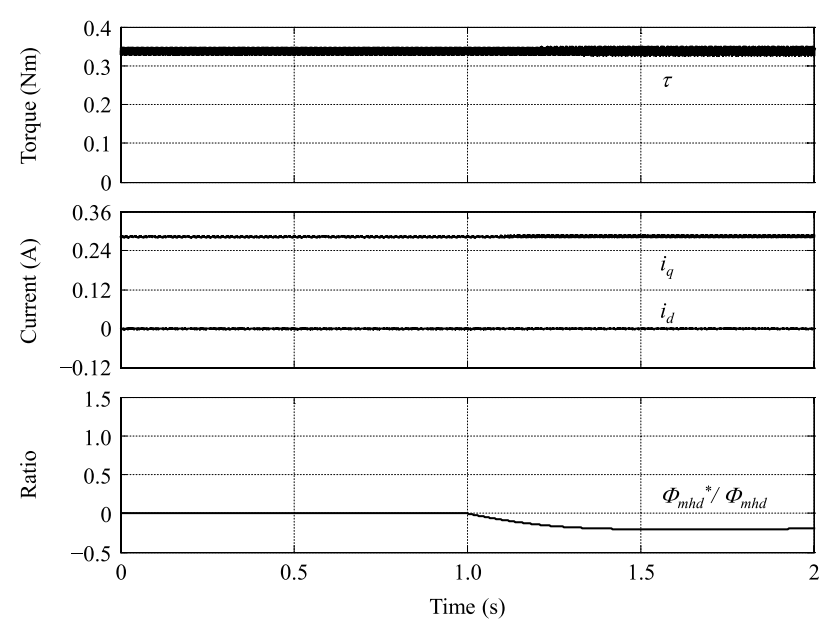

(a) Harmonic-flux estimation with d-axis current

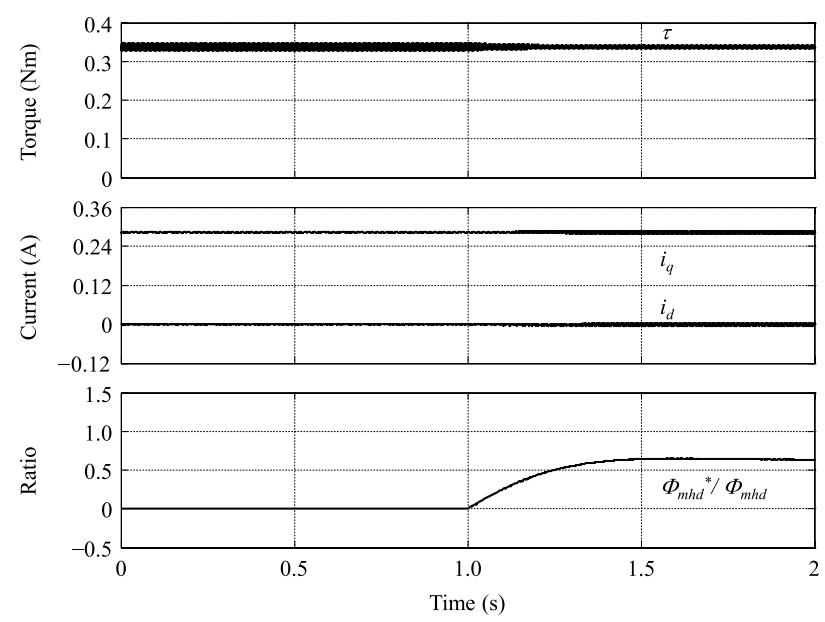

(b) Harmonic-flux estimation with q-axis current

Fig. 8. Results of the proposed control scheme by simulation with consideration of dead-time effect

from the true value, and the torque ripples increased. This is because the dead-time effect appeared prominently on the current of the non-energized axis - that is, the d-axis detected current $i_{d c}$ - and because the set values $\Phi_{m h d}{ }^{*}$ and $\Phi_{m h q}{ }^{*}$ contained errors. Figure 8(b) is the amplitude values $\Phi_{m h d}$ and $\Phi_{m h q}$ estimated using the q-axis detected current $i_{q c}$, based on Eqs. (43)-(45). Despite containing an error, the set value $\Phi_{m h d}$ converges to the true value, and the torque ripples were reduced. These results indicate that by estimating the parameters using the q-axis detected current $i_{q c}$ - the current on the energized axis - it is possible to alleviate the dead-time effect.

5.2 Experimental Investigation In an experimental investigation, it is difficult to measure an instantaneous torque containing ripple components with accuracy. For this reason, in this paper, we mounted the tested motor on the air conditioner outdoor unit and evaluated it by measuring the noise in front of the fan opening generated by ripple torque with a microphone. The microphone was installed 0.5 meters from the fan opening, and the noise level measured was corrected by A-weighting. The proposed control scheme was implemented on a 16-bit microcomputer together with the vector control unit and position sensorless control unit. The evaluation board used in the experiment is shown in Fig. 9.

Figure 10 illustrates the results obtained with the air condi- 


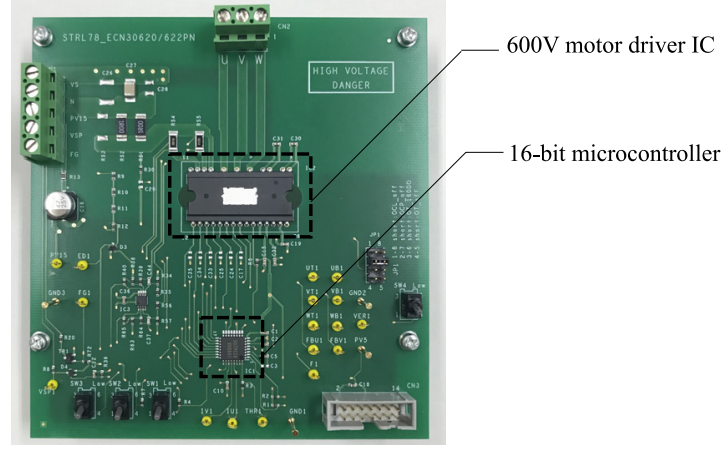

Fig. 9. PMSM driver evaluation board

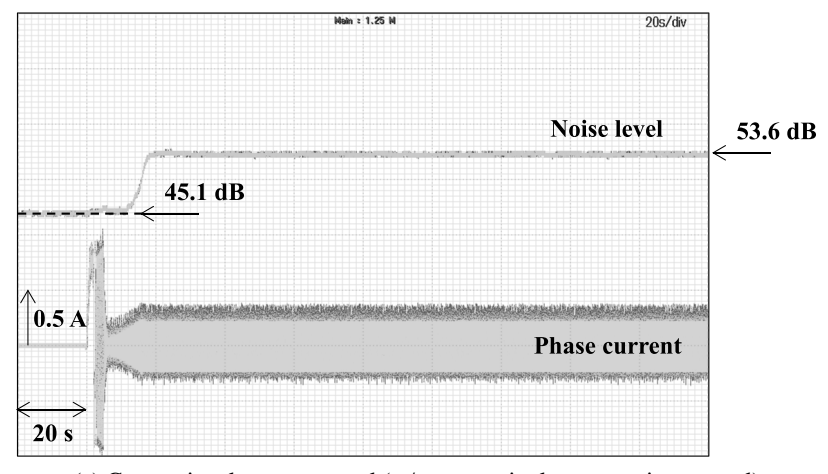

(a) Conventional vector control (w/o torque ripple suppression control)

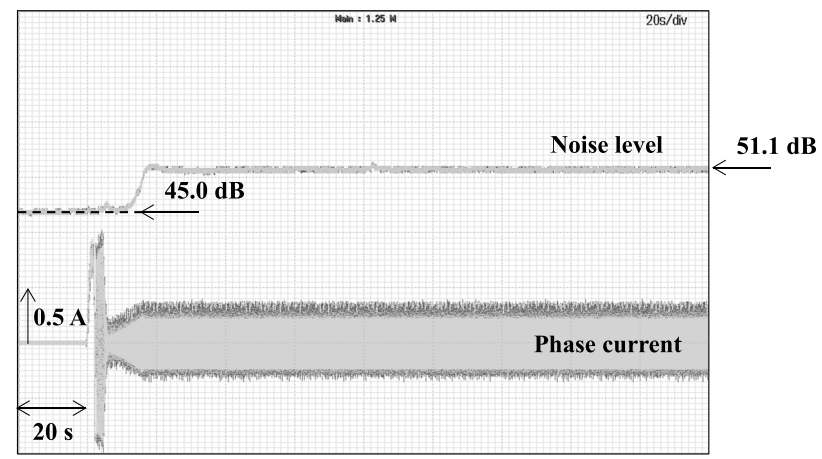

(b) Proposed control scheme (w/ torque ripple suppression control)

Fig. 10. Experimental results for measured noise level and phase current

tioner running with only the conventional vector control and when the proposed control scheme was applied. The rotational speed is $675 \mathrm{~min}^{-1}$, the d-axis current command $I_{d}{ }^{*}$ is $0 \mathrm{~A}$, the carrier frequency is $16 \mathrm{kHz}$, and the inverter DC voltage is $280 \mathrm{~V}$. These results show the operating waveform from start-up until the steady-state is reached. A comparison of the results of Figs. 10(a) and (b) indicates that the noise level in steady-state is $2.5 \mathrm{~dB}$ lower in (b), which shows that the proposed control scheme effectively reduced the noisecausing torque ripples.

Table 2 shows the results of the amplitude value, $\Phi_{m h d}$, estimated at different speed conditions. Compared to the result at $675 \mathrm{~min}^{-1}$, the accuracy at $375 \mathrm{~min}^{-1}$ and $975 \mathrm{~min}^{-1}$ deteriorated. The possible causes of estimation error include the following:

- Low-speed condition $\left(375 \mathrm{~min}^{-1}\right)$ : when the rotational speed is low, the assumption of Eq. (34) applied to the construction of the harmonic magnetic flux estimator, $\left(0 \ll \omega_{r}\right)$, does not hold, which decreases the estimation
Table 2. Results of harmonic-flux estimation at different speed conditions

\begin{tabular}{|c|c|}
\hline Motor speed & $\Phi_{\text {mhd }}^{*} / \Phi_{\text {mhd }}$ \\
\hline $375 \mathrm{~min}^{-1}$ & 1.736 \\
\hline $675 \mathrm{~min}^{-1}$ & 0.676 \\
\hline $975 \mathrm{~min}^{-1}$ & -0.316 \\
\hline
\end{tabular}

accuracy.

- High-speed condition $\left(975 \mathrm{~min}^{-1}\right)$ : as the rotational speed increased, the sampling frequency became insufficient for the ripple current, decreasing the estimation accuracy (the number of samplings per sixth-order harmonic cycle at $675 \mathrm{~min}^{-1}$ was about 19 times, but it dropped to about 13 times at $975 \mathrm{~min}^{-1}$ ).

These results indicate that there is a rotational speed range for the proposed control scheme to function effectively, and at low and high-speed rotation, it is necessary to disable the control. For example, in a case aimed at improving the quietness, it is important to confirm in advance that the range of the proposed control scheme covers the rotational speed conditions under which mechanical resonance-induced vibration noise increases. Also, low-speed rotation requires special attention because the calculation of phase difference in position sensorless control, $\Delta \theta_{c}$, becomes more susceptible to errors.

\section{Conclusion}

In this paper, we proposed a method of torque ripple suppression control with a small calculation load that can be implemented even on low-cost motor driving devices. The proposed control scheme gives compensation signals (voltage commands) in a feedforward manner and generates a ripple current to suppress torque ripples. It also extracts ripple components contained in a detected current and estimates the harmonic components of the magnetic flux based on that information. These techniques were then verified by simulation and experiment, and the results demonstrated that they can effectively reduce torque ripples. This proposed control scheme can operate using only sensors that already exist in the equipment. It also features a mechanism that partially reduces the calculation frequency, which allows it to be implemented on low-cost 16-bit microcomputers. Our future challenge is to make a theoretical analysis of the dead-time effect on the proposed control scheme and construct an effective method to compensate it.

\section{References}

( 1 ) T.M. Jahns and W.L. Soong: "Pulsating Torque Minimization Techniques for Permanent Magnet AC Motor Drives-A Review", IEEE Trans. Industrial Electronics, Vol.43, No.2, pp.321-330 (1996)

( 2 ) K. Nakamura, H. Fujimoto, and M. Fujitsuna: "Torque Ripple Suppression Control for PM Motor Considering the Bandwidth of Torque Meter", IEEJ Trans. IA, Vol.130-D, No.11, pp.1241-1247 (2010) (in Japanese)

( 3 ) T. Yamaguchi, Y. Tadano, and N. Hoshi: "Torque Ripple Suppression Control by Periodic Disturbance Observer with Model Error Correction", IEEJ Trans. IA, Vol.134-D, No.2, pp.185-192 (2014) (in Japanese)

( 4 ) S. Hattori, M. Ishida, and T. Hori: "Suppression Control Method of Vibration for PMSM Utilizing Repetitive Control with Auto-tuning Function and Fourier Transform", IEEJ Trans. IA, Vol.121-D, No.3, pp.347-355 (2001) (in Japanese) 
( 5 ) S. Shinnaka and H. Kishida: "New Simple Torque-Sensorless Torque Control for Quasi-Perfect Compensation of 6th Harmonic Torque Ripple Due to Nonsinusoidal Distribution of Back EMF of PMSM", IEEJ Trans. IA, Vol.131-D, No.8, pp.1068-1077 (2011) (in Japanese)

( 6 ) H. Hida, Y. Tomigashi, K. Ueyama, Y. Inoue, and S. Morimoto: "New Torque Estimation Method Considering Spatial Harmonics and Torque Ripple Reduction in Permanent Magnet Synchronous Motors", IEEJ Trans. IA, Vol.130-D, No.9, pp.1051-1058 (2010) (in Japanese)

( 7 ) T. Yamaguchi, Y. Tadano, and N. Hoshi: "A Study of Torque Ripple Suppression Control using a Speed Sensor with Periodic Disturbance Observer", 2012 IEEJ Industry Applications Society Conference, 3-55 (2012) (in Japanese)

( 8 ) T. Nakai and H. Fujimoto: "Harmonic Current Suppression for PMSM by Repetitive Perfect Tracking Control", IEEJ Trans. IA, Vol.128-D, No.9, pp.1083-1089 (2008) (in Japanese)

( 9 ) K. Sakamoto, Y. Iwaji, and T. Endo: "A Simplified Vector Control of Position Sensorless Permanent Magnet Synchronous Motor for Electrical Household Appliances", IEEJ Trans. IA, Vol.124-D, No.11, pp.1133-1140 (2004) (in Japanese)

\section{Appendix}

Eqs. (5) and (6) were derived as follows. First, the stator interlinkage magnetic flux generated by the permanent magnet is represented by Eq. (A1) on three-phase stationary coordinates:

$$
\begin{aligned}
{\left[\begin{array}{l}
\phi_{m u} \\
\phi_{m v} \\
\phi_{m w}
\end{array}\right]=\Phi_{m 1} \cdot\left[\begin{array}{c}
\cos \theta_{d} \\
\cos \left(\theta_{d}-\frac{2 \pi}{3}\right) \\
\cos \left(\theta_{d}+\frac{2 \pi}{3}\right)
\end{array}\right]+\Phi_{m 5} \cdot\left[\begin{array}{c}
\cos 5 \theta_{d} \\
\cos 5\left(\theta_{d}-\frac{2 \pi}{3}\right) \\
\cos 5\left(\theta_{d}+\frac{2 \pi}{3}\right)
\end{array}\right] } \\
\left.+\Phi_{m 7} \cdot\left[\begin{array}{c}
\cos 7 \theta_{d} \\
\cos 7\left(\theta_{d}-\frac{2 \pi}{3}\right) \\
\cos 7\left(\theta_{d}+\frac{2 \pi}{3}\right)
\end{array}\right] \ldots \ldots \ldots \text { (A1 }\right)
\end{aligned}
$$

$\phi_{m u}, \phi_{m v}, \phi_{m w}$ : stator interlinkage magnetic flux generated by the permanent magnet on three-phase stationary coordinates

In Eq. (A1), the harmonic components of the magnetic flux of up to the seventh order were considered.

Next, the transformation matrix that transforms the components on three-phase stationary coordinates into d-axis and q-axis components is defined by Eq. (A2):

$$
\boldsymbol{C}=\frac{2}{3} \cdot\left[\begin{array}{ccc}
\cos \theta_{d} & \cos \left(\theta_{d}-\frac{2 \pi}{3}\right) & \cos \left(\theta_{d}+\frac{2 \pi}{3}\right) \\
-\sin \theta_{d} & -\sin \left(\theta_{d}-\frac{2 \pi}{3}\right) & -\sin \left(\theta_{d}+\frac{2 \pi}{3}\right)
\end{array}\right]
$$

If the transformation matrix expressed in Eq. (A2) is applied to Eq. (A1), the stator interlinkage magnetic flux generated by the permanent magnet on d-q coordinates is represented as the next equation:

$$
\left[\begin{array}{c}
\phi_{m d} \\
\phi_{m q}
\end{array}\right]=\left[\begin{array}{c}
\Phi_{m 1} \\
0
\end{array}\right]+\left[\begin{array}{c}
\left(\Phi_{m 5}+\Phi_{m 7}\right) \cdot \cos 6 \theta_{d} \\
\left(-\Phi_{m 5}+\Phi_{m 7}\right) \cdot \sin 6 \theta_{d}
\end{array}\right]
$$

Above is how Eqs. (5) and (6) were obtained.

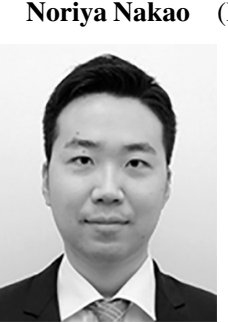

Member) received the Ph.D. degree from Shibaura Institute of Technology, Tokyo, Japan, in 2015. He was a visiting research scholar in the Wisconsin Electric Machines and Power Electronics Consortium (WEMPEC), at University of Wisconsin-Madison in 2014. He joined Hitachi, Ltd. in 2015. His research interest is control of rotating machines. Dr. Nakao is a member of the Institute of Electrical Engineers of Japan (IEEJ).

Kazuaki Tobari (Member) received the Ph.D. degree from Tohoku

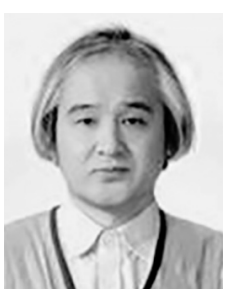
University, Miyagi, Japan, in 2016. He joined Hitachi Research Laboratory, Hitachi, Ltd. in 1985. His research interest is control of induction motors and permanent magnet synchronous motors. Dr. Tobari is a member of the Institute of Electrical Engineers of Japan (IEEJ).

Tomohiro Sugino (Member) was born in 1976. He received the M.S. degree from Kyushu Institute of Technology, Fukuoka, Japan, in 2001. He joined Hitachi, Ltd. in 2001 and has been with Hitachi Power Semiconductor Device, Ltd. since 2013. His professional interests are design and control of inverter ICs. Mr. Sugino is a member of the Institute of Electrical Engineers of Japan (IEEJ)

Yoshiki Ito (Member) was born in 1971. He received the M.S.

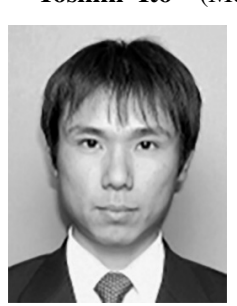
degree from Niigata University, Niigata, Japan, in 1996. He joined Hitachi Industry \& Control Solutions, Ltd. (formerly Hitachi Process Computer Engineering, Inc.) in 1996. His professional interests are design and control of inverter ICs. Mr. Ito is a member of the Institute of Electrical Engineers of Japan (IEEJ)

Mitsuhiro Mishima (Member) was born in 1973. He received the

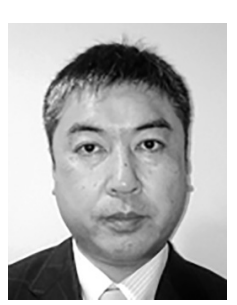
M.S. degree from Iwate University, Iwate, Japan, in 1998. He joined Hitachi Industry \& Control Solutions, Ltd. (formerly Hitachi Process Computer Engineering, Inc.) in 1998. His professional interests are design and control of inverter ICs. Mr. Mishima is a member of the Institute of Electrical Engineers of Japan (IEEJ)

Daisuke Maeda

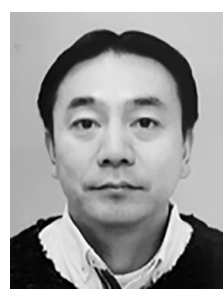

(Member) was born in 1976. He received the B.S. degree from Yokohama National University, Kanagawa, Japan, in 1999. He joined Hitachi, Ltd. in 1999 and has been with Hitachi Power Semiconductor Device, Ltd. since 2013. His professional interest is business planning of power semiconductor devices. Mr. Maeda is a member of the Institute of Electrical Engineers of Japan (IEEJ). 